\title{
Michael GOMEZ, African Dominion: a New History of Empire in Early and Medieval West Africa
}

\section{Hadrien Collet}

\section{OpenEdition}

\section{Journals}

Édition électronique

URL : https://journals.openedition.org/ccm/5080

DOI : $10.4000 / \mathrm{ccm} .5080$

ISSN : 2119-1026

\section{Éditeur}

Centre d'études supérieures de civilisation médiévale/Université de Poitiers

\section{Édition imprimée}

Date de publication : 1 septembre 2020

Pagination : $172-176$

ISBN : 978-2-490783-06-9

ISSN : 0007-9731

\section{Référence électronique}

Hadrien Collet, « Michael gomez, African Dominion: a New History of Empire in Early and Medieval West Africa », Cahiers de civilisation médiévale [En ligne], 250-251 | 2020, mis en ligne le 01 septembre 2020, consulté le 30 novembre 2022. URL : http://journals.openedition.org/ccm/5080 ; DOI : https://doi.org/ $10.4000 / \mathrm{ccm} .5080$

\section{cc) (i) (9)}

Creative Commons - Attribution - Pas d'Utilisation Commerciale - Pas de Modification 4.0 International - CC BY-NC-ND 4.0

https://creativecommons.org/licenses/by-nc-nd/4.0/ 
Michael Gomez, African Dominion: a New History of Empire in Early and Medieval West Africa, Princeton, Princeton University Press, 2018.

Avec African Dominion, Michael Gomez présente l'ambition de susciter le débat et de ramener l'histoire de l'Afrique de l'Ouest médiévale dans la conversation historiographique relative à l'histoire des empires en particulier, et l'histoire mondiale en général. C'est peu dire que, depuis sa sortie en 2018, le livre n'a pas laissé indifférent. L'a. a lui-même participé à des tables rondes avec d'autres universitaires, dont certaines ont été publiées, tandis que des recensions ont également émergé ( Review roundtable », The American Historical Review, 124-2, 2019, p. 581-594 avec comme communiquants: Ghislaine Lydon, Ousmane Kane, Shamil Jeppie, Paulo Fernand o de Moraes Farias, avec réponse de M. Gomez; Amir Syed, « African Dominion: a New History of Empire in Early and Medieval West Africa, written by Michael A. Gomez ", Islamic Africa, 10, 2019, p. 225-227. Une autre table ronde a pris place pendant le congrès de l'African Studies Association [ASA] en novembre 2019 mais ses actes ne sont pas encore publiés. Les communiquants étaient Bruce Hall, Chouki El Hamel, Ousmane Kane et Jan Jansen. J'ai, par ailleurs, déjà évoqué la question des empires et de la tradition impériale dans African Dominion dans une autre contribution : Hadrien Collet, « Empires of Reflections: Searshing for Medieval Empires and Imperial Tradition in Historiographies of West Africa ", The Journal of African History, sous presse) Il est possible d'observer deux tendances globalement parallèles quant à la réception du livre. D'un côté, le milieu des chercheurs spécialistes du Moyen Âge africain s'est montré assez sévère, d'un autre côté, la critique historienne états-unienne, africaniste ou plus généraliste, a salué un livre nécessaire et indispensable, décernant deux prix au livre. Dernièrement, l'African Studies Association (ASA) nord-américaine l'a par ex. récompensé fin novembre 2019 de son book award. Il ne s'agira pas ici de répéter ces critiques, auxquelles j'agrée en grande partie, le lecteur les trouvera à partir des notes. Néanmoins, avant d'aborder 
les pages se rapportant spécifiquement à la période s'étirant du $\mathrm{X}^{\mathrm{e}}$ au XIII ${ }^{\mathrm{e}} \mathrm{s}$., j'aimerais présenter le livre dans son ensemble ainsi que quelques réflexions sur les frictions causées depuis sa sortie, qui peuvent s'interpréter selon moi comme un malentendu ou plutôt un décalage entre la manière dont l'éditeur a vendu et promu African Dominion et ce que porte réellement l'ouvrage.

Le livre couvre environ un millénaire d'histoire, du milieu du $\mathrm{VI}^{\mathrm{e}}$ s. à l'année 1591, date de la chute de l'empire Songhay suite à sa conquête par le Maroc. M. Gomez propose une structure en trois grandes parties («Early Sahel and Savannah », « Imperial Mali » et « Imperial Songhay ») et onze chapitres dont les 2 à 5 seront discutés ici. D'un point de vue général, le livre n'introduit pas de nouvelles sources mais s'appuie davantage sur les corpus existant, principalement sur le Corpus of Early Arabic Sources for West African History, publié pour la première fois par Nehemia Levtzion et John F. P Hopkins en 1981 ( Cambridge/Londres, Cambridge University Press [Fontes historiae africanae, Series Arabica, 4], 1981), ainsi que sur un socle de savoir sur la région connu et publié depuis longtemps. Toutefois, et c'est là l'une des principales critiques qui a été formulée à l'égard du livre, $\mathrm{M}$. Gomez intègre très peu à sa réflexion les deux dernières décennies de recherche qui ont pourtant révolutionné les études médiévales sur l'Afrique de l'Ouest, ayant entraîné des tournants historiographiques et épistémologiques tant dans les domaines des manuscrits arabes ouest-africains, de l'épigraphie ou de la littérature orale. (Pour avoir un aperçu d'ensemble de cette dynamique : Landscapes, Sources and Intellectual Projects of the West African Past: Essays in Honour of Paulo Fernando de Morae Farias, T. Green, B. Rossi [dir.], Leyde, Brill, 2018). Le champ disciplinaire des études médiévales ouest-africaines connaît en effet depuis deux décennies un renouveau, particulièrement en ce qui concerne le Sahel occidental qui occupe l'essentiel de l'attention de la recherche. Pourtant, dans la courte notice présentant le livre, les presses universitaires de Princeton le décrivent comme pionnier « the first on this period of the region's history in a generation " (https://press. princeton.edu/books/hardcover/9780691177427/ african-dominion). C'est ici que survient le premier décalage, entre l'annonce dans le sous-titre d'une " nouvelle histoire » et une certaine prise de distance avec un champ d'études qui s'est considérablement renouvelé au $\mathrm{XXI}^{\mathrm{e}} \mathrm{s}$.

Il serait ainsi plus juste de dire qu'African Dominion est le premier ouvrage à proposer une grande histoire narrative depuis longtemps. Cette approche a un coût, puisque la connaissance de l'Afrique subsaharienne à la période médiévale se fait par le biais de sources arabes externes à la région, tandis que les sources arabes endogènes s'inscrivent, elles, dans une temporalité postmédiévale. En outre, les sources sont extrêmement fragmentaires et variées, et, difficulté supplémentaire, véhiculent parfois des discours ou des régimes de vérité différents et difficilement réconciliables. Il existe donc dans notre courant d'étude une certaine réticence, voire résistance, à revenir vers une histoire du grand récit, d'autant plus que les grandes synthèses avaient émergé en premier pendant la période coloniale dans un style très positiviste, avant la constitution de corpus et tout travail critique sur les documents. En somme, notre discipline s'était construite à l'envers. Depuis les années 1960, d'autres formes de narrativité ont ainsi été continuellement explorées (dès 1961 par Raymond MAuny avec sa thèse Tableau géographique de l'Ouest africain au Moyen Âge d'après les sources écrites, la tradition et l'archéologie [Dakar, Ifan]; plus récemment, François-Xavier FaUVELLEAYMAR a fait le pari d'une narrativité par le fragment dans son Rhinocéros d'or, histoires du Moyen Âge africain [Paris, Alma, 2013]). Adopter le modèle d'un récit quasi continu courant sur la période médiévale implique donc nécessairement de revenir à une lecture plus littérale et moins déconstructiviste des sources tout en envisageant qu'un registre de sources a vocation à intervenir pour combler les manques et les silences d'un autre. L'écueil de prendre les sources au pied de la lettre, dans lequel tombe souvent l'a., est d'ailleurs un reproche qui revient souvent dans les comptes rendus (voir par ex. : A. Syed [art. cit. supra], p. 226-227). A vrai dire le travail philologique et critique du document n'intéresse, à quelques exceptions près, que très peu l'a. Aussi, pour comprendre la démarche de M. Gomez, il faut peut-être moins considérer African Dominion comme l'étude historique classique d'un spécialiste en dialogue avec son champ que comme un essai d'histoire, un essai de réinterprétation.

Cette considération implique à minima de chercher une thèse principale que viendrait appuyer une certaine lecture de l'histoire déployée sur cet objet d'étude. Le prologue, qui fait office d'introduction, la met en exergue à la dernière phrase : « The result is a wholly new interpretation of West Africa's early and medieval history, facilitating its relocation from the periphery to the center of world history » (p. 7). Passer par une histoire des empires pour donner voix au chapitre à l'Afrique de l'Ouest dans la conversation 
de l'histoire mondiale. C'est une nécessité il est vrai. Si des ratés spectaculaires se sont produits récemment dans l'histoire mondiale des empires où l'Afrique était absente, il faut signaler toutefois que l'idée n'est pas entièrement nouvelle. Rien qu'en France par ex., de nombreux projets collectifs d'histoire mondiale ont témoigné d'une intégration toujours plus croissante de l'histoire de l'Afrique avant la fin du XIX ${ }^{\mathrm{e}} \mathrm{s}$. (L'histoire du monde au $X v^{e}$ siècle, P. Boucheron [dir.], Paris, Fayard, 2009; tout récemment : L'exploration $d u$ monde : une autre histoire des grandes découvertes, R. Bertrand [dir.], Paris, Seuil, 2019). Si African Dominion renoue en quelque sorte avec une vieille histoire politique et dynastique, sa vision du social emprunte au puissant moment historiographique qui scande aujourd'hui l'histoire de l'Afrique (mais pas seulement) aux États-Unis construisant son questionnaire à partir des catégories d'ethnicité (ethnicity), de racialité sociale (race), d'identité (identity) et de genre (gender). Ici réside la véritable nouveauté du livre. Mais peut-on appliquer ces concepts et réflexions, forgés sur des terrains de recherche et des temporalités postmédiévaux à la période médiévale au Sahel occidental? Dans le cadre de l'esclavage et de l'islam, par ex., les assignations raciales et identitaires prirent une importance considérable dans les débats savants de la région puisque d'elles pouvaient dépendre l'asservissement de groupes entiers. Toutefois ces débats apparaissent surtout dans la littérature arabe postmédiévale, à un moment où la diversité chromatique des esclaves prit peu à peu fin.

Disons un dernier mot enfin sur la thématisation de l'histoire du Sahel occidental à la période médiévale. Depuis le début du Xx ${ }^{\mathrm{e}}$ s., le Moyen Âge soudanien est défini par un âge impérial pendant lequel se seraient succédé trois grandes hégémonies, celles du Ghāna, du Māli et du Songhay. Cette lecture politique de la période a fini par la subsumer entièrement par le biais du chrononyme " temps des grands empires » ou « temps des empires Ghāna, Māli, Songhay ». Toutefois, comme nous allons en discuter ci-après, ce modèle commence tout juste à être remis en cause.

Au début du $\mathrm{X}^{\mathrm{e}} \mathrm{s}$., une mosaïque de royaumes et de cité-États coexistaient au Sahel occidental dont la complexité géopolitique nous échappe en grande partie en raison du manque de sources. Parmi ces États, se distinguaient Gao à l'est, Ghāna au centre et Takrūr à l'extrême ouest. Le chap. 2 («Early Gao ») est consacré au royaume de Gao. Dans l'introduction de ce chapitre, M. Gomez annonce que les chapitres à venir (chap. 3 : «The Kingdoms of Ghana : Reform along the Senegal River »; chap. 4 : « Slavery and Race Imagined in Bilād As-Sūdān ») fonctionnent comme une seule séquence. Dans l'introduction à ces trois chapitres donc, M. Gomez avance un certain nombre d'idées dans une formulation assez représentative de sa méthode. Il propose d'abord une réévaluation du statut de Gao, puis de l'expansion almoravide comme un mouvement réformateur décisif concernant l'islamisation de la région. Ce moment marqua notamment, selon lui, une transformation de Ghāna qui aboutit non pas à son affaiblissement, comme on l'a longtemps cru, mais déboucha sur un nouvel apogée. Enfin, cette nouvelle expression d'un islam qu'il perçoit comme plus radical obligerait à réexaminer la question de l'esclavage. Concernant l'utilisation des sources, principale pierre d'achoppement relevée par la critique, M. Gomez entend faire feu de tout bois, utilisant sans distinction les chroniques de Tombouctou du milieu du XVII ${ }^{\mathrm{e}} \mathrm{s}$, les sources médiévales externes, les données archéologiques, et les inscriptions épigraphiques. « Further contesting the secondary literature is the conclusion that these very different sources are far more harmonious than has been represented » (p. 20). Il existe pourtant des travaux récents extrêmement argumentés et documentés qui démontrent exactement l'inverse et surtout en expliquent les raisons (l'étude la plus emblématique de ce tournant critique vis-à-vis des sources : Paulo Fernando de MoraEs FARIAs, Arabic Medieval Inscriptions from the Republic of Mali: Epigraphy, Chronicles and Songhay-Tuāreg History, Oxford, Oxford University Press [Fontes historiae africanae, 4], 2003). M. Gomez s'en affranchit ici en mettant à distance le travail de ses collègues, une constante dans le livre, faisant souvent de cette " littérature secondaire » un bloc essentialisé et uniforme. Pourtant, rappelons-le, son travail, de nature réinterprétative, s'appuie largement sur cette dernière, ce qui comprend également les corpus des sources narratives traduites passées au filtre subjectif et situé de ses devanciers. Toutefois, cette littérature secondaire n'est pas entièrement considérée comme un pôle problématique à mettre de côté. Le rapport que l'a. entretient avec elle varie selon qu'il peut fondre telle ou telle étude dans son grand récit sans en affecter la logique et la linéarité.

L'histoire qu'il restitue du royaume de Gao, dont la capitale a été absorbée par la ville contemporaine de Gao, s'appuie sur des sources connues depuis longtemps et s'avère assez classique. Ce qui est nouveau est qu'il fait du royaume le point de départ («Gao as West Africa's Starting Point », p. 20) de la grande histoire médiévale au Sahel occidental en lieu et place de Ghāna, challengeant le modèle traditionnel des successions hégémoniques Ghāna-Māli-Songhay. Son 
argumentation aurait pu être appuyée par la découverte à Gao par les équipes de Mamadou Cissé et Shoichiro Takezawa du seul palais fortifié construit en pierre connu actuellement dans la région, et de son trésor archéologique témoignant d'une remarquable accumulation de richesse à cette époque (Mamadou Cissé et Shoichiro TAKEZAwA, « Discovery of the Earliest Royal Palace in Gao and its Implications for the History of West Africa », Cahiers d'études africaines, 208, 2012, p. 813-844).Toutefois, on aurait aimé qu'il aille plus loin dans la déconstruction de ce modèle. En substituant Ghāna par Gao, il ne fait qu'y ajouter un nouveau segment, alors qu'il serait plus juste de dire que les transformations sociales et politiques favorisées par les nouvelles connexions longue distance permises par l'islam et le commerce transsaharien ont affecté concomitamment toute la façade sahélienne. Plutôt que de chercher un unique point de départ à une nouvelle forme médiévale du politique, il faudrait davantage considérer le phénomène comme affectant tout le Sahel, l'immensité de l'espace considéré faisant saillir une dynamique d'ensemble multisituée.

Les chapitres couvrant la période du $\mathrm{X}^{\mathrm{e}}$ au XIII ${ }^{\mathrm{e}} \mathrm{s}$. souffrent d'un double problème. Premièrement, toutes les sources sont mises sur le même plan, sans considération de leur contexte de production, des différences chronologiques ou de leur nature. Même au sein d'un genre spécifique, le manque de travail critique sur les sources conduit l'a. à des affirmations erronées. C'est par ex. le cas concernant le royaume de Ghāna, dont M. Gomez découpe l'histoire en plusieurs séquences. D'abord « païen », le royaume se convertit à l'islam dans un contexte de guerre et de troubles marqués au moment de la conquête almoravide au milieu du XI ${ }^{\mathrm{e}} \mathrm{s}$, avant un nouvel optimum. Dans la première partie du XIII ${ }^{\mathrm{e}}$ s., Ghāna entame un déclin irréversible. Pour éclairer ce contexte, M. Gomez précise alors : « there is a hint of regression in the sources, with ancestral religion resurgent, reinforced by the observation that, save for the Muslims, 'its inhabitants go naked', with women covering their pubic areas with beads of glass or shells, or bones if poorer $\gg($ p. 40). Il renvoie en note à al-Qazwīnī, auquel ce passage fait référence, qui écrit v. 1275. Or, ce dernier ne fait que reprendre des auteurs comme Ibn Faqīh (v. 903) et Yaqūt (v. 1220) qui compile lui-même des sources plus anciennes. Le passage ne renvoie donc absolument pas à des réalités historiques du XIII $\mathrm{s}$.

Secondement, hasard du calendrier éditorial, la parution d'African Dominion s'est produite dans un contexte où plusieurs publications d'importance ont également vu le jour : Sedrata. Histoire et archéologie d'un carrefour du Sahara médiéval : à la lumière des archives inédites de Marguerite van Berchem, C. Aillet, P. Cressier et S. Gilotte (dir.), Madrid, Casa de Velázquez (Collection de la Casa de Velázquez, 161), 2017; Essouk - Tadmekka, an Early Islamic Trans-Saharan Market Town, S. NixON (éd.), Leiden/Boston (Mass), Brill (Journal of African Archaeology Monograph Series, 12), 2017 ; Landscapes, Sources and Intellectual Projects of the West African Past: Essays in Honour of Paulo Fernando de Moraes Farias, T. Green et B. Rossi (éd.), Leiden/Boston, Brill, 2018; L'Afrique ancienne, De l'Acacus au Zimbabwe. 20000 avant notre ère-XVIII siècle (2018) (F.-X. FAuVELle [dir.], Paris, Belin [Mondes anciens], 2018), pour ne citer que les ouvrages. On trouvera dans ces études une histoire de la région du $\mathrm{X}^{\mathrm{e}}$ au XIII ${ }^{\mathrm{e}} \mathrm{s}$. plus à jour.

M. Gomez fait du XI ${ }^{\mathrm{e}}$ s., avec la conquête almoravide et la conversion à l'islam des élites sahéliennes, un tournant nécessitant de reconsidérer le syntagme « Bilād al-Sūdān » et la question de l'esclavage en lien avec les débats sur la race, dont il donne sa définition: " This study understands race as referring to the culturally orchestrated, socially sanctioned disaggregation and reformulation of the human species into broad, hierarchical categories reflecting purported respective levels of capacity, propensity, and beauty, and in ways often tethered to phenotypic expression » (p. 56).

Le terme « Bilād al-Sūdān », littéralement pays des « Sūdān », mot désignant dans son sens premier les populations noires, ou identifiées comme telles, de l'Afrique subsaharienne, fut très tôt adopté par la géographie arabe médiévale. Les idées défendues par M. Gomez sont que l'essor du commerce transsaharien s'est accompagné d'un processus concomitant associant toujours plus étroitement les Sūdān avec l'état servile via le commerce des esclaves (p. 47 et 53), que la notion de " Bilād al-Sūdān » fut une « racialisation de l'espace » (p. 54) et que l'islam a entraîné l'adoption de nouvelles conceptions raciales néfastes pour les populations noires toujours plus étroitement associées avec arriérisme et servilité (p. 55). Pour appuyer son argumentation, il passe en revue les sources narratives qui font montre en effet d'un certain nombre de stéréotypes à l'encontre des Sūdān, agrégeant des genres allant de la littérature des merveilles aux débats islamologiques des savants médiévaux sur le mythe de Cham. Il apporte néanmoins quelques concessions en pointant les appartenances multiples d'un même individu du point de vue ethnolinguistique, religieux, régional, des activités économiques, etc., qui, par leur combinaison, 
pouvaient « reroute the process of categorizing the darker-skinned » (p. 56). Mais cela n'affecte en rien son idée générale, et les dissonances pouvant le contredire sont gommées, comme la question des Touaregs à la peau sombre («darker skinned Tuaregs ») simplement «blancs » en vertu de leur statut («by virtue of status », p. 57). S'il est vrai que cette question tient une place importante dans l'histoire de l'Afrique de l'Ouest, la période médiévale présente en revanche une spécificité qui mériterait de nuancer le propos, la « racialisation » des esclaves devenant prégnante surtout à la toute fin du Moyen Âge et dans les siècles postmédiévaux. Pour la période qui nous concerne ici $\left(\mathrm{X}^{\mathrm{e}}-\mathrm{XIII}{ }^{\mathrm{e}} \mathrm{s}.\right)$, plusieurs exemples peuvent être apportés pour montrer que la « question raciale », loin de porter un processus de réification inéluctable, était plastique, labile et dynamique, et ne peut être résumée à une lutte dialectique ou de pouvoir entre deux pôles, l'un bìdān (blanc) et l'autre sūdān (noir). Sur la question du toponyme Bilād al-Sūdān, tout d'abord, l'on observe assez rapidement dans les sources qu'une acception géographique cohabite avec une définition plus « ethniciste » avant que la première ne prenne le dessus sur la seconde. Ainsi, par ex., les sources juridiques de Kairouan du XI ${ }^{\mathrm{e}}$ s. font commencer le Bilād al-Sūdān, sur l'axe commercial oriental du Sahel occidental, avec la ville de Tadmekka, à l'extrême nord du Sahel donc, majoritairement habitée par des Berbères. Cela va d'ailleurs dans le sens de la remarque de M. Gomez concernant Ahmmad Bābā, le grand savant berbère de Timbuktu du tournant du $\mathrm{XVI}^{\mathrm{e}}$ s. qui est désigné au Maroc comme al-Sūdān̄̄ (littéralement originaire du Soudan), disant que sa nisba (nom d'origine) pouvait simplement se référer à sa région d'origine, malgré la tentation d'y voir un «phenotypic descriptor» (p. 52). En outre, l'une des spécificités de la période médiévale était que l'on trouvait, du Sahara à la savane, autant des esclaves blancs (berbères, turcs) que noirs. Cela s'explique à la fois par une pratique de l'esclavage, surtout domestique, antérieure à l'arrivée de l'islam, mais également par le processus d'islamisation en cours, qui faisait que des « poches » de populations non monothéistes pouvaient être asservies par les musulmans, dans un cadre religieux donc, et non dans celui d'une partition raciale. Il faut ajouter à cela que les sources attestent d'une circulation dans les deux sens, organisée par les États sahéliens (pour le pèlerinage à La Mecque par ex.) ou non. Les sociétés du Maghreb et du Moyen-Orient, surtout Le Caire, étaient donc habituées à voir des petites communautés d'hommes noirs libres dans leur ville, phénomène qui s'accroît $\mathrm{au} \mathrm{XIII}^{\mathrm{e}} \mathrm{s}$. et qui devient encore plus important les deux siècles suivants. Il n'y avait donc pas forcément un réflexe de les associer immédiatement à la servilité comme ce fut davantage le cas par la suite. D'ailleurs, à côté des stéréotypes péjoratifs, pouvaient également cohabiter des descriptions extrêmement laudatives, comme celle que l'on trouve sur la beauté du visage de l'empereur du Māli Mansa Musa, suite à son passage au Caire en 1324. Enfin, les attributions du statut de bìdān et sūdān au Sahel occidental dépendaient moins à cette époque de la couleur de peau que d'autres facteurs, comme l'activité économique (agriculteur ou éleveur) et le lieu de résidence (steppe/désert ou vallées fluviales). Ainsi, comme l'a montré P. F. de Moraes Farias, un groupe pouvait apparaître, à deux intervalles de temps différents, d'abord Bīdān puis Sūdān, et inversement, et naviguer entre ces catégories (Paulo Fernando de MoRA ES FARIAS, « Touareg et Songhay, histoires croisées, historiographies scindées », Berbères ou Arabes? Le tango des spécialistes, H. Claudot-Hawad [dir.], Paris/Aix-en-Provence, Éd. Non Lieu/Iremam, 2006, p. 225-262). Il faut donc nuancer fortement le propos de M. Gomez pour rendre à la période médiévale sa spécificité et sa complexité, résister à la tentation de voir les transformations sociales et politiques des siècles postmédiévaux, dramatiques pour les populations noires, comme ayant nécessairement des racines historiques beaucoup plus profondes, et se rappeler qu'il y avait au Sahel occidental des États forts, reconnus comme tels par l'Islam médiéval, qui imposaient leur loi et leur volonté.

Au final, le projet d'African Dominion d'offrir un grand récit pouvant rivaliser avec l'histoire mondiale des empires semble prendre le pas sur le reste. L'essai de réinterprétation est fortement marqué, comme nous le disions en introduction, par l'historiographie étatsunienne actuelle et ses préoccupations très contemporaines. Le livre est exigeant, écrit dans un langage châtié, voire parfois abstrait. Toutefois, le style très soutenu et la puissance du récit ne parviennent pas à dissimuler l'aporie d'une démarche refusant de réellement engager le dialogue scientifique avec le champ d'études dans lequel s'inscrit pourtant de facto le livre.

Hadrien Collet

Institut français d'archéologie orientale 